\title{
Facial paralysis caused by malignant skull base neoplasms
}

\author{
Sam J. Marzo, M.D., John P. Leonetti, M.D., and Guy Petruzzelli M.D., Ph.D. \\ Center for Cranial Base Surgery, Loyola University, Chicago, Illinois
}

\begin{abstract}
Object. Bell palsy remains the most common cause of facial paralysis. Unfortunately, this term is often erroneously applied to all cases of facial paralysis.

Methods. The authors performed a retrospective review of data obtained in 11 patients who were treated at a university-based referral practice between July 1988 and September 2001 and who presented with acute facial nerve paralysis mimicking Bell palsy. All patients were subsequently found to harbor an occult skull base neoplasm. A delay in diagnosis was demonstrated in all cases. Seven patients died of their disease, and four patients are currently free of disease.

Conclusions. Although Bell palsy remains the most common cause of peripheral facial nerve paralysis, patients in whom neoplasms invade of the facial nerve may present with acute paralysis mimicking Bell palsy that fails to resolve. Delays in diagnosis and treatment in such cases may result in increased rates of mortality and morbidity.
\end{abstract}

KEY WORDS • skull base tumor • malignancy • facial paralysis • Bell palsy

Bell palsy can be defined as an acute, unilateral, lower motor neuron facial paresis or paralysis, in which no other cause can be identified. ${ }^{3}$ Although it remains the most common cause of facial nerve paralysis, various intracranial, intratemporal, extratemporal, skull base, or systemic diseases can also be responsible (Table 1). Although Bell palsy has been defined as idiopathic, there is now good evidence to implicate the activation of herpes simplex virus near the geniculate ganglion as the cause of this disorders. ${ }^{1,6}$ Cases in which temporal bone trauma or intratemporal infections cause facial nerve paralysis can often be correctly identified when the patients present for examination. In cases of occult intratemporal, extratemporal, or skull base malignancies, however, patients can also present with acute facial paresis or paralysis resembling Bell palsy. Many such patients present to otolaryngologists in a delayed fashion, which may result in a further delay in diagnosis. We treated 11 patients in whom occult skull base malignancies had been incorrectly diagnosed as Bell palsy. We present the clinical manifestations, evaluation, management, and outcome of these cases.

\section{CLINICAL MATERIAL AND METHODS}

We performed a retrospective analysis of all otolaryngological patients who presented with facial paralysis for

Abbreviations used in this paper: $\mathrm{CT}=$ computerized tomography; $\mathrm{MR}=$ magnetic resonance. examination to our institution between July 1988 and September 2001. There were six men and five women who ranged in age from 62 to 77 years (mean age 69.4 years). During this 13-year period, there were approximately 19,200 new patient visits, 320 of whom were for facial paresis or paralysis. Eleven of these 320 cases met the criteria of this study - namely, presentation with facial paralysis mimicking Bell palsy, that was found to be caused by an occult malignancy. The records of these patients were retrospectively reviewed. All patients underwent a complete otoneurological and cranial nerve examination, as well as neuroimaging that included fine-cut CT scanning and MR imaging. Surgical therapy, including a superficial or complete parotidectomy, neck dissection, and/or some form of temporal bone resection, was performed in all patients. Radiotherapy was undertaken postoperatively in nine patients and preoperatively in one patient (the standard dose was 50-60 Gy, administered in divided fractions over 5-6 weeks).

\section{RESULTS}

We retrospectively reviewed the cases of 11 patients who presented with facial paralysis. Presenting symptoms are listed in Table 2. Pain, which was present in all patients, was the most common symptom. The most common presenting sign was facial paralysis (Table 2). Facial paralysis manifested in five patients in an acute fashion $(<24$ hours), in three patients in a subacute fashion $(24$ 
TABLE 1

Differential diagnosis of facial nerve paralysis

\begin{tabular}{l}
\hline Cause \\
\hline infectious \\
Bell palsy \\
herpes zoster oticus \\
acute otitis media \\
mastoiditis \\
necrotizing external otitis \\
skull base osteomyelitis \\
Lyme disease \\
other \\
traumatic \\
basilar skull fracture \\
birth injury \\
penetrating temporal bone trauma \\
parotid injury \\
iatrogenic \\
neoplastic \\
facial nerve neuroma \\
facial nerve hemangioma \\
carcinoma (primary or metastatic \& intra- or extratemporal) \\
jugular glomus \\
vestibular schwannoma \\
meningioma \\
metastatic lesion \\
inflammatory \\
cholesteatoma \\
sarcoidosis \\
Wegener granulomatosis \\
other \\
congenital \\
\hline
\end{tabular}

48 hours), and in three patients in a delayed fashion $(>48$ hours). The second most common presenting sign was a mass located in the parotid gland. Based on clinical and neuroimaging findings, tumor origins were found to be temporal bone (four patients), parotid gland (five patients), and combined temporal bone and parotid gland (two patients). All tumors were determined to be malignant (Table 3). The surgical therapies are summarized in Table 4.

The follow-up period ranged from 6 months to 11 years (mean 7.4 years) after diagnosis. Table 5 lists treatment re-

TABLE 2

Presenting symptoms \& signs

\begin{tabular}{lc}
\hline \hline Symptom/Sign & No. of Patients \\
\hline symptom & 11 \\
pain & 3 \\
numbness & 3 \\
otalgia & 2 \\
voice change & 2 \\
dysphagia & 1 \\
deafness & \\
sign & 11 \\
facial paralysis & 7 \\
mass & 3 \\
otorrhea & \\
cranial nerve deficit & 3 \\
V & 1 \\
VII & 2 \\
X & \\
\hline
\end{tabular}

TABLE 3

Summary of malignant lesions

\begin{tabular}{cc}
\hline \hline Tumor Histology & No. of Patients \\
\hline adenocarcinoma & 5 \\
squamous cell carcinoma & 3 \\
adenoid cystic carcinoma & 2 \\
osteogenic sarcoma & 1 \\
\hline
\end{tabular}

sults. Patients were followed for at least 6 months, or until death. Patients usually died of locally advanced or systemic disease. At the time of this writing seven patients had died of their disease and four were alive without disease. All patients presented in a delayed fashion (Table 6). The mean delay in diagnosis was 5 months.

\section{ILLUSTRATIVE CASES}

\section{Case 1}

This 71-year-old man presented in January 1998 with a 2-year history of left-sided preauricular numbness and delayed left facial nerve paralysis ( $>48$ hours); left otalgia initially began in March 1997. After a diagnosis of Bell palsy was reached, he had been referred to us for treatment. Clinical examination revealed a complete left facial nerve paralysis, whereas MR imaging demonstrated enhancement of the left facial nerve within the geniculate and tympanic segments. After obtaining a transcanal biopsy sample of the abnormal tympanic segment of the left facial nerve, examination showed poorly differentiated adenocarcinoma. The patient later underwent a temporal bone resection and parotidectomy, after which full-course radiotherapy was undertaken. Six months later he developed diplopia with a left abducent nerve paralysis, and recurrent disease was found within the left cavernous sinus (Fig. 1). A metastatic survey was positive for liver and chest involvement, and the patient died several months later.

\section{Case 2}

This 64-year-old man presented to us in December 1991 with a 6-month history of complete right-sided facial nerve paralysis and otalgia. After a diagnosis of Bell palsy was reached, he was referred to us for treatment. Clinical examination revealed a complete right facial nerve paralysis with a 3-cm right tail of parotid mass. Results of fineneedle aspiration were consistent with a diagnosis of poorly differentiated squamous cell carcinoma. The patient

TABLE 4

Summary of surgical treatments*

\begin{tabular}{cc}
\hline \hline \multicolumn{1}{c}{ Op } & No. of Patients \\
\hline subtotal TBR + parotidectomy & 5 \\
parotidectomy + MRND & 2 \\
TBR + parotidectomy + MRND & 3 \\
translabyrinthine craniotomy & 1 \\
\hline
\end{tabular}

* MRND $=$ modified radical neck dissection; TBR $=$ temporal bone resection. 
TABLE 5

Treatment results

\begin{tabular}{lc}
\hline \hline \multicolumn{1}{c}{ Outcome } & No. of Patients \\
\hline dead of local disease & 2 \\
dead of metastatic disease & 4 \\
dead of local \& metastatic disease & 1 \\
alive w/out disease & 4 \\
\hline
\end{tabular}

underwent a total parotidectomy, neck dissection, facial nerve sacrifice, and a lateral temporal bone resection. $\mathrm{He}$ later underwent a static facial nerve suspension in which gold weight implantation was performed. He received postoperative radiotherapy. He remained free of disease until 1994 when he presented with recurrent right-sided neck disease for which he underwent a right radical neck dissection, trapezius flap reconstruction, and stereotactic radiotherapy. He subsequently developed chest metastases in February 1996 and died the following month.

\section{Case 3}

This 76-year-old man presented in May 1991 with a complete right-sided facial nerve paralysis and a painful, chronic draining right ear. He had apparently been told he had a right-sided Bell palsy. In 1977 he had sustained a burn injury on the right side of his face and pinna. Subsequently, a squamous cell carcinoma developed in the right temporal bone, and, at an outside institution, he had undergone two mastoid surgeries and full-course radiotherapy. Physical examination revealed an infected right mastoid cavity in which the bone was exposed and a complete right facial nerve paralysis. An MR imaging study demonstrated findings consistent with osteoradionecrosis (Fig. 2). He underwent hyperbaric oxygen therapy. Thereafter, a right temporal bone resection, pedicled trapezius flap reconstruction, a static facial nerve suspension, and gold weight implantation were performed. He remains without evidence of recurrent disease.

\section{DISCUSSION}

In cases of neoplastic invasion of the facial nerve patients may present with acute facial paralysis or paresis mimicking Bell palsy. Bell palsy remains a diagnosis of exclusion and is best made after a thorough otolaryngological examination demonstrates no evidence of occult malignancy. The advent of MR imaging has allowed identification of characteristics suggestive of a viral inflammation of the facial nerve.,10 The usual pattern seen on MR imaging in cases of Bell palsy is enhancement of the

TABLE 6

Delay before correct diagnosis of malignant skull base neoplasm

\begin{tabular}{cc}
\hline \hline Delay in Mos & No. of Patients \\
\hline $1-3$ & 5 \\
$3-6$ & 4 \\
$6-9$ & 1 \\
$>9$ & 1 \\
\hline
\end{tabular}

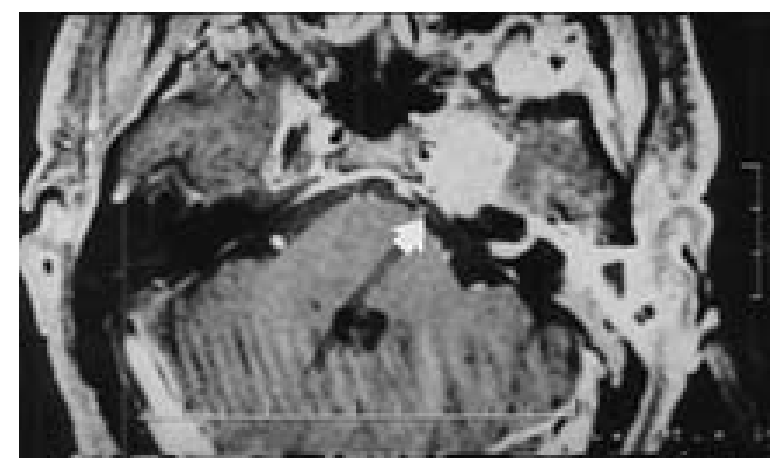

Fig. 1. Case 1. Magnetic resonance image demonstrating recurrent disease within the left cavernous sinus (arrow).

nerve within the meatal, geniculate, and sometimes tympanic segments, which may last for 4 months. ${ }^{9}$ This enhancement may persist while facial nerve recovery is occurring. ${ }^{7}$ Neoplastic invasion of the facial nerve may show enlargement of the nerve or enhancement in only one segment. ${ }^{9}$ Although MR imaging shows great promise in aiding in the management of patients with facial nerve paralysis, it cannot always differentiate between a Bell palsy and neoplasm; nor can its findings be used to predict recovery completely. ${ }^{5}$ Furthermore, normal-appearing features demonstrated on an MR image do not exclude the presence of a malignant facial nerve lesion. In one study of 486 patients with unilateral facial nerve paralysis, malignant tumors with facial nerve involvement were found 19 patients. In eight of these 19 patients initial MR imaging demonstrated normal anatomy. ${ }^{4}$ The authors thus argued that facial nerve paralysis without evidence of regeneration at 6 months is the manifestation of a malignant lesion and merits surgical exploration regardless of MR imaging findings. Transmastoid and transparotid facial nerve exposure should be performed first and followed by a middle fossa facial nerve exposure and decompression.

Malignant invasion of the facial nerve is an ominous finding. Patients with Ramsay Hunt syndrome, which is

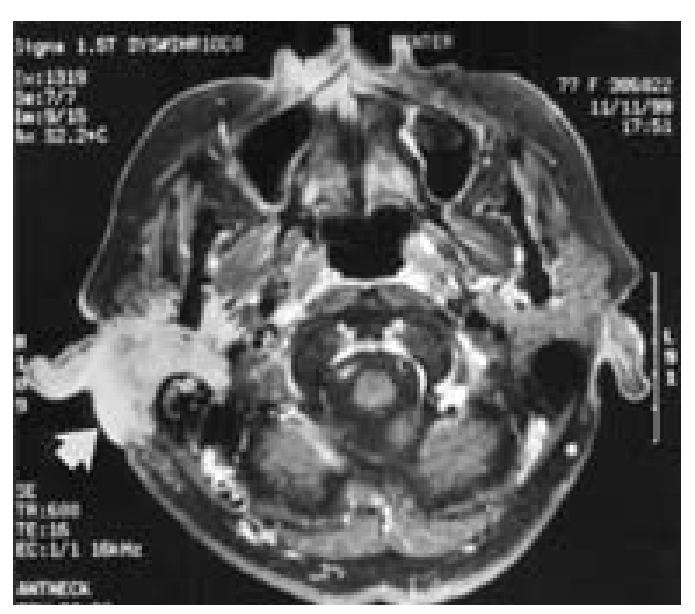

Fig. 2. Magnetic resonance image revealing chronic osteomyelitis of the left temporal bone (arrow). 


\section{S. J. Marzo, J. P. Leonetti, and G. Petruzzelli}

characterized by the triad of otalgia, facial paralysis, and vesicular rash, can experience severe persistent pain; in these cases diagnosis is aided by the identification of the classic vesicular pattern on the skin and pinna. When no vesicles are present, the finding of pain in conjunction with facial nerve paralysis might suggest an invasion of the nerve by a malignant neoplastic process. Other symptoms such as painful chronic otorrhea might suggest a neoplasm of the temporal bone. In cases of parotid masses pain and facial paralysis may also suggest a malignant process.

The overall poor survival seen in our patients with malignant facial nerve paralysis is in agreement with that reported by other authors who have studied malignant parotid tumors. In analyzing 110 cases of malignant parotid tumors in which patients underwent radiotherapy and surgery between 1970 and 1986, Pedersen, et al., ${ }^{8}$ found no survivors after 8 years in patients who presented with a primary facial nerve palsy. A delay in diagnosis, which is unfortunately common when these disease processes are misdiagnosed, can also be a factor because time allows for potential continued growth of the neoplasm, which may adversely affect local or regional control of disease.

Although the numbers of affected patients in this study and other studies are small due to the rarity of this condition, it will be interesting to see if modern skull base surgery can increase the survival rate of patients with malignant facial paralysis. The presence of malignant facial nerve paralysis is a poor prognostic sign, and in the aforementioned study performed by Pedereson, et al. ${ }^{8}$ no survivors were reported after 8 years. This study commenced before the era of modern skull base surgery. It remains to be seen if skull base surgery can improve survival in patients with malignant skull base tumors. Our opinion is that modern skull base surgery will lead to improved local and regional control of disease, which may result in increased long-term survival rates. Although we cannot prove that the delay in the diagnosis in the aforementioned patients resulted in adverse outcome, we believe that the sooner a malignant facial nerve neoplasm is diagnosed, the better the chance for local and regional control of disease and, perhaps, survival.

The only means by which to diagnose these tumors sooner is through clinical assessment and judgment. Magnetic resonance imaging and CT scanning are helpful, although their findings are not definitive. Most patients with Bell palsy do not experience pain, and some facial nerve recovery, usually within 6 weeks to 3 months, is observed in all. It is our opinion that patients in whom painful facial paralysis is unresolved after 3 months should undergo contrast-enhanced MR imaging of the head, internal auditory canals, and parotid gland with contrast, temporal bone CT scanning, and surgical exploration of the facial nerve based on neuroimaging findings. If the neuroimaging studies demonstrate normal findings, the surgeon should be prepared to perform a total facial nerve exploration.

\section{CONCLUSIONS}

Neoplastic invasion of the facial nerve may mimic signs and symptoms of Bell palsy. We have reviewed the cases of 11 patients who presented in such a fashion and in whom occult temporal bone or parotid malignancies were found. A delay in diagnosis, which may have adversely affected survival, was a factor present in all cases. Survival was poor. Seven patients died of locally advanced or systemic disease, and four patients remain alive, free of disease. Facial or referred pain occurring with a facial nerve paralysis suggests neoplastic invasion of the facial nerve. The prognosis in such cases may be grave, and a diligent search for a malignant lesion of the facial nerve should be performed. Furthermore, through continued education we will hopefully disabuse those who persist in misapplying the term Bell palsy. With future improvements in diagnostic methods and viral detection, we may one day be able to diagnose and label correctly each cause of each facial nerve paralysis.

\section{References}

1. Burgess RC, Michaels L, Bales JF Jr, et al: Polymerase chain reaction amplification of herpes simplex viral DNA form the geniculate ganglion of a patient with Bell's palsy. Ann Otol Rhinol Laryngol 103:775-779, 1994

2. Daniels DL, Czervionke LF, Millen SJ, et al: MR imaging of facial nerve enhancement in Bell palsy or after temporal bone surgery. Radiology 171:807-809, 1989

3. Gantz BJ, Rubinstein JT, Gidley P, et al: Surgical management of Bell's palsy. Laryngoscope 109:1177-1188, 1999

4. Jungehuelsing M, Sittel C, Fischbach R, et al: Limitations of magnetic resonance imaging in the evaluation of perineural tumor spread causing facial nerve paralysis. Arch Otolaryngol Head Neck Surg 126:506-510, 2000

5. Millen SJ, Daniels D, Meyer G: Gadolinium-enhanced magnetic resonance imaging in facial nerve lesions. Otolaryngol Head Neck Surg 102:26-33, 1990

6. Murakami S, Mizobuchi M, Nakashiro Y, et al: Bell palsy and herpes simplex virus: identification of viral DNA in endoneurial fluid and muscle. Ann Intern Med 124:27-30, 1996

7. Murphy TP: MRI of the facial nerve during paralysis. Otolaryngol Head Neck Surg 104:47-51, 1991

8. Pedersen D, Overgaard J, Sogaard H, et al: Malignant parotid tumors in 110 consecutive patients: treatment results and prognosis. Laryngoscope 102:1064-1069, 1992

9. Schwaber MK, Larson TC III, Zealear DL, et al: Gadoliniumenhanced magnetic resonance imaging in Bell's palsy. Laryngoscope 100:1264-1269, 1990

10. Schwaber MK, Zealear D, Netterville JL, et al: The use of magnetic resonance imaging with high-resolution CT in the evaluation of facial paralysis. Otolaryngol Head Neck Surg 101: 449-458, 1989

Manuscript received March 19, 2002.

Accepted in final form April 22, 2002

Address reprint requests to: Sam J. Marzo, M.D., Department of Otolaryngology-Head and Neck Surgery, Loyola University Medical Center, 2160 South First Avenue, Maywood, Illinois 60153. email: smarzo@luc.edu. 